\title{
Review Article \\ Clinical Pharmacology of Chemotherapy Agents in Older People with Cancer
}

\author{
Xiaoye He, ${ }^{1,2}$ Stephen J. Clarke, ${ }^{3}$ and Andrew J. McLachlan ${ }^{2,4}$ \\ ${ }^{1}$ Department of Geriatrics, Zhongshan Hospital, Fudan University, Shanghai 200032, China \\ ${ }^{2}$ Faculty of Pharmacy, University of Sydney, NSW 2006, Australia \\ ${ }^{3}$ Sydney Medical School and Royal North Shore Hospital, University of Sydney, NSW 2006, Australia \\ ${ }^{4}$ Centre for Education and Research on Ageing, Concord Hospital, NSW 2139, Australia \\ Correspondence should be addressed to Andrew J. McLachlan, andrew.mclachlan@sydney.edu.au
}

Received 27 March 2011; Accepted 23 June 2011

Academic Editor: Victoria Cogger

Copyright ( 2011 Xiaoye He et al. This is an open access article distributed under the Creative Commons Attribution License, which permits unrestricted use, distribution, and reproduction in any medium, provided the original work is properly cited.

Populations around the world are aging, and the associated increase in cancer incidence has led to the recognition of the importance of geriatric oncology. Chronological age is a poor determinant of pharmacological response to cancer chemotherapy agents. Age-associated changes in physiology and organ function have a significant impact on the clinical pharmacology of cancer chemotherapy agents used in cancer treatment. Altered response to medicines in older people is a consequence of changes in body composition, organ function, concomitant pathophysiology, multiple medications, genetic determinants of drug response, and patient's clinical status. These issues highlight the need to individualize the management of cancer in the older people with consideration of age-related changes in the clinical pharmacology of cancer drugs, analgesics, and adjunctive therapies.

\section{Introduction}

The continued growth in the proportion of older people in many developed countries has resulted in a large number of older adults with cancer. Cancer is now the second most common cause of death, and approximately half of all cancers occur in the population aged 65 years and older [1]. There is an increasing number of older patients who require the management of cancer. Understanding and defining the optimum use of cancer chemotherapy agents in older cancer patients in the adjuvant, curative, and palliative settings is a very pressing and timely issue in oncology. However, until recently, the area of geriatric oncology has been relatively ignored and there has been less research undertaken than in other areas of oncology which has resulted in a lack of evidence-based guidelines for the treatment of older cancer patients. A significant challenge in providing cancer care to older people is that aging is an individualized and heterogeneous process with variable effects on the clinical pharmacology of drugs used in the management of cancer. This has important implications for choosing the optimal drug regimen, selecting a safe and effective initial, dose and undertaking appropriate monitoring strategies in managing older people with cancer.

The aim of this paper is to identify and discuss the factors which affect the clinical pharmacology of chemotherapy agents in older people with cancer and the medical management of these patients.

\section{Factors Affected the Clinical Pharmacology of Chemotherapy in Older Cancer Patients}

\subsection{Age-Related Changes in Clinical Pharmacology}

2.1.1. Pharmacokinetics. Studies investigating age-related changes in the pharmacokinetics of cancer chemotherapy agents have provided conflicting evidence [2]. While some studies report age-related differences in the pharmacokinetics of cancer chemotherapy agents, most studies have reported no significant differences based on patient chronological age per se, or only minor differences in the pharmacokinetics of chemotherapy agents in older people. However, many of the older patients included in these studies have been selected on the basis of good performance status and 
normal organ function and thus may not be representative of patients treated in routine practice. Changes observed in the pharmacokinetics of drugs in older people (when compared to younger people) result from age-related changes in physiology and organ function and/or comorbid disease [3].

Pharmacokinetic assessments in patients should incorporate consideration of factors affecting drug absorption, distribution, metabolism, and excretion.

Drug absorption can be affected by age-related changes in the physiology of the gastrointestinal tract such as decreased splanchnic circulation, reduced gastric motility and secretion, and reduction in the absorptive surface area for drugs $[4,5]$. However, there are relatively few examples of drugs affected by these changes which result in clinically relevant alterations in the extent of drug absorption. Typically the rate of absorption may be reduced in older people but the extent of absorption (associated with bioavailability) remains largely unaffected [3] in older patients. Age-associated changes in gastrointestinal physiology and the potential effects on drug absorption may come into sharper focus with the trend for increased use of oral chemotherapeutic drugs. This is an important area for future investigation.

Aging is associated with changes in body composition, including a reduction in total body water, protein stores, and lean body mass with an increase in proportion of body fat [6]. Decreased intracellular water leads to a reduced volume of distribution ( $\mathrm{V}$ ) for hydrophilic drugs that primarily distribute to body water, while the relative increase in adipose tissue in older people can result in an increase in the volume of distribution of lipid-soluble drugs. This may result in a reduction in the maximum concentration achieved after a dose and prolongation of the terminal half-life of an agent in older people when compared to younger patients [7]. The reduction in serum albumin concentration in older adults results in an increase in the unbound fraction of some drugs, which may have important implications [8] for the distribution of drugs bound to albumin. Studies had shown that low serum albumin concentrations in malnourished older patients with advanced cancer resulted in a low clearance of highly albumin-bound drugs which, in turn, caused increased free drug concentration and contributed to unexpected toxicity [9].

Clearance, a measure of the efficiency of drug elimination, is the most important pharmacokinetic variable as it is the main determinant of maintenance dose rate for multiple dosing drug regimens and of drug exposure (i.e., area-under-the-concentration-time curve, AUC) after a single dose. Drug metabolism has the greatest impact on clearance for most drugs (and in turn, exposure) and is therefore likely to significantly influence a patient's beneficial and adverse response at a given dose [10]. The liver is the principal site of drug metabolism. Age-related changes in the liver include decreased liver weight, reduced hepatic blood flow, and reduced amount and activity of cytochrome P450 enzymes, in association with an overall reduction in the metabolic capacity of the liver in older people $[4,5]$. Many chemotherapy agents, including the taxanes (paclitaxel and docetaxel), cyclophosphamide, and vinca alkaloid drugs (vincristine, vinblastine, and vinorelbine), and molecular targeted drugs are metabolized by cytochrome P450 enzymes, especially CYP3A4, and predominantly undergo hepatic metabolism and clearance [7]. There is clear evidence that the liver sinusoidal endothelial cells in the aging liver undergo pseudocapillarization such that the sieving function of liver is significantly reduced with age [11]. This agerelated change in the liver has implications for highly protein bound drugs and large macromolecules or proteinbased therapeutic agents (such as liposomal therapies, e.g., liposomal doxorubicin) with the potential for a reduction in hepatic clearance due to reduced access to the hepatocytes. Biliary excretion is a key hepatic elimination pathway for numerous drugs and their metabolites. While this is a major pathway of elimination of commonly used drugs such as the anthracyclines (doxorubicin, epirubicin, and daunorubicin), it appears to be unaffected by the age of the patient and the associated changes in physiology [12].

There is increasing recognition that inflammation associated with advanced cancer and frailty in older people has a significant effect on the regulation of drug metabolising enzymes and transporters $[13,14]$. It has been demonstrated in both clinical and preclinical studies that elevated plasma concentrations of inflammatory proteins are associated with reduced hepatic drug clearance and increased toxicity from chemotherapy [15]. Inflammation has the potential to downregulate drug metabolism and transporter pathways further complicating the impact of advanced cancer and frailty on the clinical pharmacology of cancer chemotherapy agents [16-18]. Given the impact of inflammation on drug metabolism, it is, therefore, not unreasonable to hypothesize that the level of inflammatory markers in blood might correlate with variations seen in the metabolism of anticancer drugs. However, this relationship should be evaluated in prospective trials incorporating pharmacokinetic analyses of cytotoxic drugs.

Many drugs and metabolites are renally eliminated from the body. Renal clearance is the result of glomerular filtration, renal secretion (mediated by transporters), and tubular reabsorption. A reduction in glomerular filtration rate (GFR) is almost universal with age, and it has been reported that there is a decrease in the GFR of approximately $1 \mathrm{~mL} / \mathrm{minute}$ for every year over the age of 40 years [19]. The kidneys' ability to appropriately concentrate or dilute urine and excrete water and electrolytes is also impaired with aging $[5,20]$. This decline in renal function may be associated with an increased risk of toxicity for renally excreted cytotoxic agents and their metabolites, such as platinum compounds, alkylating agents, capecitabine, purine analogues, antimetabolites, camptothecins, and etoposide [7]. It is recommended to estimate the creatinine clearance ( $\mathrm{CrCl}$, as a marker of a person's renal function) with the formula of Cockcroft and Gault [21] in all patients with age of 50 years or more to guide the drug and dosage selection $[3,22,23]$. While there is now a range of equations which have been used to estimate a person's GFR based on serum creatinine concentration, the CrCL remains the best and most reliable metric to estimate renal function for the determination of drug dose regimens for renally excreted medicines [3, 21, 23]. 
2.1.2. Pharmacodynamics. Most of the age-related differences in drug response observed in older cancer patients are in the realm of pharmacodynamics and manifest as decrements in end-organ function [3]. Age-related changes in effector system function, organ function, and impaired homeostatic control result in age-related changes in pharmacodynamics. End-organ response is affected by physiological changes that occur with increasing age in the absence of pathology [23] or in the context of concomitant multiple pathophysiological changes. For example, the diminished homeostatic reserve in older people [3], especially those that are frail, leads to a greater impact of haematological toxicities from cancer chemotherapy agents.

The pharmacodynamic changes of aging may affect both the efficacy and the toxicity of many antineoplastic agents. The increased prevalence of MDR-1 in acute myeloid leukemia [24], increased resistance to apoptosis in follicular lymphoma [25], increased adhesion of neoplastic cells to stroma in multiple myeloma [26], decreased tumour growth fraction [27], tumour cell anoxia, and abnormal chemotherapy targets [28] are all examples of age-associated changes in pharmacodynamics that impact the efficacy of chemotherapy agents in older cancer patients.

The impaired homeostatic control associated with aging also increases the risk of short- and medium-term complications of cancer chemotherapy, including myelosuppression [29], acute cardiomyopathy [30], peripheral and central neuropathy [31] and mucositis [32], as well as long-term complications [33-36] such as chronic cardiomyopathy from anthracyclines, increased incidence of myelodysplasia (MDS), and acute myeloid leukemia (AML).

2.2. Comorbidity. The occurrence of comorbid medical conditions such as diabetes, heart disease, hypertension, arthritis, and lung disease affects many older patients [37]. The likelihood of an older person experiencing a chronic illness increases rapidly with age. In cancer patients, comorbidity limits treatment options, negatively affects treatment tolerance, and influences the presence and severity of symptoms and other complications [38]. For example, more attention should be paid to patients with ischemic heart disease when they are treated with fluoropyrimidines as a previous study has demonstrated that patients with a history of cardiac disease, particularly coronary artery disease, were significantly more susceptible to 5-FU cardiotoxicity when compared to patients without this medical history [39]. Patients with impaired lung function will face increased risk of pulmonary toxicity from bleomycin. Giving cisplatin to patients with poor cardiac function will increase the risk of the onset of heart failure due to the requirement for prehydration with large volumes of fluids. Comorbidity is also associated with poorer survival, increasing both cancer and noncancer related mortality [40, 41]. The treatment of comorbid conditions during cancer treatment may result in an increased likelihood of drug interactions. In addition, cancer treatments, including chemotherapy, radiation therapy, and other supportive care drugs, may exacerbate comorbid conditions [23].
2.3. Polypharmacy. The obvious consequence of comorbidity is the concurrent use of multiple medications. Of all the factors that are most consistently associated with adverse drug reactions, inappropriate or unnecessary polypharmacy has been considered the most important. Using multivariate analysis, studies have demonstrated that the principal contributor to adverse drug reactions in older people is inappropriate polypharmacy [23]. The harms associated with polypharmacy include increased risks of adverse drug reactions, drug interactions, increased healthcare costs, and errors in patient adherence to therapy [23]. Older age, comorbidity, recent hospitalization, female gender, depression, number of treating doctors, and practitioner characteristics are the main risk factors for polypharmacy [42]. Some medicines place older people at a significantly higher risk of adverse effects and serious drug interactions [42]. The Beers Criteria provide an important starting point to improve prescribing by limiting the use of medicines that pose a high risk of adverse effects in older people $[42,43]$.

\section{Medical Management in Older People with Cancer}

3.1. Comprehensive Geriatric Assessment. A comprehensive geriatric assessment (CGA) approach, including identification of frailty, can assist oncologists in identifying older patients who are more likely to develop severe toxicity during cancer treatment [44]. Components of the GCA, which involves a multidimensional evaluation to determine the overall health status of an older adult, have been used effectively to guide treatment planning and decision making in older adults with cancer. The CGA determines which patients may benefit from and tolerate standard cancer treatment including identifying those most likely to derive benefit from a palliative treatment approach [45].

Although the CGA is a useful tool in the assessment of older people with cancer, this approach is time-consuming and is only indicated in selected patients [46, 47]. Some clinical oncologists have used an abbreviated tool such as the VES-13 to screen patients who should go on to a full CGA [48]. In addition to this, the National Comprehensive Cancer Network (NCCN) has developed useful guidelines for pretreatment screening to determine which patients, such as those aged 70 years or more and whose hemoglobin levels are under $12 \mathrm{mg} / \mathrm{dL}$, who may need a more comprehensive assessment [49].

3.2. Treatment Strategies. Medical oncologists have gradually started to appreciate that the approach to cancer chemotherapy that is adopted for otherwise healthy adults cannot automatically be applied to older patients with agerelated changes in physiology, reduced homeostatic reserve, and comorbid medical problems. Numerous questions need to be addressed prior to commencing chemotherapy in older patients including which anticancer agents should we choose in each particular circumstance? Should we use single agents or combination chemotherapy? Should we use oral or intravenous administration? These issues must be taken into consideration by the oncologists when determining treatment strategies in older cancer patients [50]. Chemotherapy 
regimens that equally optimise efficiency, tolerance, and compliance should be preferred in older cancer patients. However, the strategy of using and applying the best clinical evidence to guide treatment selection is a considerable challenge as older patients are often excluded from clinical trials.

3.3. Efficiency and Toxicity. Although standard treatment regimens may be safe and effective in older adults, the treatment of cancer in older patients requires an individualized approach. As discussed, older patients are more prone to toxicity from chemotherapeutic agents, due to age-related changes in both pharmacokinetics and pharmacodynamics [51]. The common treatment toxicities of particular concern when caring for older adults with cancer are myelosuppression, mucositis, cardiotoxicity, neurotoxicity, and musculoskeletal side effects [52].

\section{Frailty}

Frail older people are defined as those with an excess reduction in lean body mass and mobility, poor tolerance to therapy and fatigue, presence of geriatric syndromes, and/or the presence of multiple comorbidities [53]. Frailty reflects a common view of aging, indicating a critically exhausted functional reserve [54]. The recognition of frailty is particularly useful in planning cancer chemotherapy in older individuals. It may allow the practitioner to identify patients who are most at risk of chemotherapy-induced adverse outcomes and to implement a strategy to monitor and prevent these complications [53]. The frail older cancer patient needs a different approach to chemotherapy prescription. Sarcopenia, inflammation, and poor nutritional status are associated with frailty in older people $[52,54,55]$. The factors have the potential to significantly affect the clinical pharmacology of cancer chemotherapy agents. Simple pharmacological palliation of symptoms, rather than administration of potentially toxic chemotherapy, may be the preferred option in frail older people; however, the rationale for this option may need careful, sympathetic, and lengthy explanation to the patient and their relatives. Relatively less toxic single agent therapies may be the best choice in many solid tumour types, but may not be effective in rapidly growing tumours such as high-grade non-Hodgkin lymphomas or acute myeloid leukaemia [50].

\section{Summary and Outlook for the Future}

Older people represent an increasing proportion of the population and are a heterogeneous patient group at high risk for developing cancer. Age-related physiological changes may have a considerable impact on the pharmacokinetic and pharmacodynamic properties of cancer chemotherapy agents. For anticancer drugs, which have a low therapeutic index even in optimal circumstances, pharmacological changes can result in dramatic consequences, such as excessive increases in drug concentrations that produce severe and even life-threatening toxicities when standard dosing regimens are employed. In general, chronological age per se is not a contraindication to receiving cancer chemotherapy [56]. Comorbidity and poor functional status, which may be present in a significant number of the older patient population, are the main limiting factors. The key strategy is to focus on the clinical pharmacology of cancer chemotherapy agents and to individualises treatments which can be achieved by understanding the nature and extent of age-related changes in physiology and organ function. Older cancer patients, especially those with comorbidities, have been historically omitted from clinical trials resulting in study populations that are selected for their fitness and thus not representative of typical older cancer patients. It still remains a challenge to tailor and deliver the most beneficial treatments for those over the age of 65 years, taking into account comorbidities and physiologic reserves. Fortunately, there are clinical trials within various cooperative groups directed toward the development of effective and safe treatment strategies for the older people with cancer. These considerations will have important implications for training the next generation of healthcare professionals to meet the future needs [57] of older people living with cancer.

\section{Acknowledgment}

The authors acknowledge the financial support of the University of Sydney Ageing \& Alzheimer's Research Foundation (AARF).

\section{References}

[1] L. Balducci and M. Extermann, "Cancer and aging: an evolving panorama," Hematology/Oncology Clinics of North America, vol. 14, no. 1, pp. 1-16, 2000.

[2] A. Hurria and S. M. Lichtman, "Pharmacokinetics of chemotherapy in the older patient," Cancer Control, vol. 14, no. 1, pp. 32-43, 2007.

[3] A. J. McLean and D. G. Le Couteur, "Aging biology and geriatric clinical pharmacology," Pharmacological Reviews, vol. 56, no. 2, pp. 163-184, 2004.

[4] S. M. Lichtman, "Guidelines for the treatment of elderly cancer patients," Cancer Control, vol. 10, no. 6, pp. 445-453, 2003.

[5] R. Sawhney, M. Sehl, and A. Naeim, "Physiologic aspects of aging: impact on cancer management and decision making, part I," Cancer Journal, vol. 11, no. 6, pp. 449-460, 2005.

[6] I. Elmadfa and A. L. Meyer, "Body composition, changing physiological functions and nutrient requirements of the elderly," Annals of Nutrition and Metabolism, vol. 52, supplement 1, pp. 2-5, 2008.

[7] S. M. Lichtman and M. K. Boparai, "Anticancer drug therapy in the older cancer patient: pharmacology and polypharmacy," Current Treatment Options in Oncology, vol. 9, no. 2-3, pp. 191-203, 2008.

[8] J. M. Butler and E. J. Begg, "Free drug metabolic clearance in elderly people," Clinical Pharmacokinetics, vol. 47, no. 5, pp. 297-321, 2008.

[9] I. Walter-Sack and U. Klotz, "Influence of diet and nutritional status on drug metabolism," Clinical Pharmacokinetics, vol. 31, no. 1, pp. 47-64, 1996.

[10] G. R. Wilkinson, "Drug therapy: drug metabolism and variability among patients in drug response," New England Journal of Medicine, vol. 352, no. 21, pp. 2211-2221, 2005. 
[11] D. G. Le Couteur, R. Fraser, S. N. Hilmer, L. P. Rivory, and A. J. McLean, "The hepatic sinusoid in aging and cirrhosis: effects on hepatic substrate disposition and drug clearance," Clinical Pharmacokinetics, vol. 44, no. 2, pp. 187-200, 2005.

[12] S. Monfardini, "Prescribing anti-cancer drugs in elderly cancer patients," European Journal of Cancer, vol. 38, no. 18, pp. 23412346, 2002.

[13] K. Bandeen-Roche, Q. L. Xue, L. Ferrucci et al., "Phenotype of frailty: characterization in the Women's health and aging studies," Journals of Gerontology-Series A Biological Sciences and Medical Sciences, vol. 61, no. 3, pp. 262-266, 2006.

[14] S. X. Leng, Q. L. Xue, J. Tian, J. D. Walston, and L. P. Fried, "Inflammation and frailty in older women," Journal of the American Geriatrics Society, vol. 55, no. 6, pp. 864-871, 2007.

[15] K. A. Slaviero, S. J. Clarke, and L. P. Rivory, "Inflammatory response: an unrecognised source of variability in the pharmacokinetics and pharmacodynamics of cancer chemotherapy," Lancet Oncology, vol. 4, no. 4, pp. 224-232, 2003.

[16] R. E. Hubbard, M. S. O, B. L. Calver, and K. W. Woodhouse, "Plasma esterases and inflammation in ageing and frailty," European Journal of Clinical Pharmacology, vol. 64, no. 9, pp. 895-900, 2008.

[17] E. T. Morgan, K. B. Goralski, M. Piquette-Miller et al., "Regulation of drug-metabolizing enzymes and transporters in infection, inflammation, and cancer," Drug Metabolism and Disposition, vol. 36, no. 2, pp. 205-216, 2008.

[18] K. W. Renton, "Regulation of drug metabolism and disposition during inflammation and infection," Expert Opinion on Drug Metabolism and Toxicology, vol. 1, no. 4, pp. 629-640, 2005.

[19] T. Wasil and S. M. Lichtman, "Clinical pharmacology issues relevant to the dosing and toxicity of chemotherapy drugs in the elderly," Oncologist, vol. 10, no. 8, pp. 602-612, 2005.

[20] A. E. Luckey and C. J. Parsa, "Fluid and electrolytes in the aged," Archives of Surgery, vol. 138, no. 10, pp. 1055-1060, 2003.

[21] D. G. Waller, J. S. Fleming, B. Ramsay, and J. Gray, "The accuracy of creatinine clearance with and without urine collection as a measure of glomerular filtration rate," Postgraduate Medical Journal, vol. 67, no. 783, pp. 42-46, 1991.

[22] D. R MacDonald, "Neurotoxicity of chemotherapeutic agents," in The Chemotherapy Source Book, M. C. Perry, Ed., pp. 666-679, Williams \& Wilkin, Baltimore, Md, USA, 1992.

[23] S. N. Hilmer, A. J. McLachlan, and D. G. Le Couteur, "Clinical pharmacology in the geriatric patient," Fundamental and Clinical Pharmacology, vol. 21, no. 3, pp. 217-230, 2007.

[24] M. Melchert and J. Lancet, "Acute myeloid leukemia in the elderly," in Blood Disorders in the Elderly, L. Balducci, W. B. Erhsler, and G. DeGaetano, Eds., pp. 237-255, Cambridge University Press, Cambridge, UK, 2008.

[25] J. Campisi, "Aging and cancer cell biology, 2008," Aging Cell, vol. 7, no. 3, pp. 281-284, 2008.

[26] T. J. Alekshun and M. Alsina, "Multiple myeloma," in Blood Disorders in the Elderly, L. Balducci, W. B. Erhsler, and G. DeGaetano, Eds., pp. 272-289, Cambridge University Press, Cambridge, UK, 2008.

[27] M. G. Daidone, D. Coradini, G. Martelli, and S. Veneroni, "Primary breast cancer in elderly women: biological profile and relation with clinical outcome," Critical Reviews in Oncology/Hematology, vol. 45, no. 3, pp. 313-325, 2003.

[28] L. Balducci, "Cancer chemotherapy in the older person," in Blood Disorders in the Elderly, L. Balducci, W. B. Erhsler, and G. DeGaetano, Eds., pp. 225-236, Cambridge University Press, Cambridge, UK, 2008.
[29] N. M. Kuderer, D. C. Dale, J. Crawford, and G. H. Lyman, "Impact of primary prophylaxis with granulocyte colonystimulating factor on febrile neutropenia and mortality in adult cancer patients receiving chemotherapy: a systematic review," Journal of Clinical Oncology, vol. 25, no. 21, pp. 31583167, 2007.

[30] J. D. Floyd, D. T. Nguyen, R. L. Lobins, Q. Bashir, D. C. Doll, and M. C. Perry, "Cardiotoxicity of cancer therapy," Journal of Clinical Oncology, vol. 23, no. 30, pp. 7685-7696, 2005.

[31] M. Walker and O. Ni, "Neuroprotection during chemotherapy: a systematic review," American Journal of Clinical Oncology, vol. 30, no. 1, pp. 82-92, 2007.

[32] S. T. Sonis, "Pathobiology of oral mucositis: novel insights and opportunities," Journal of Supportive Oncology, vol. 5, no. 9, supplement 4, pp. 3-11, 2007.

[33] M. Schaapveld, O. Vissor, M. J. Lowman et al., "Risk of new primary non-breast cancers after breast cancer treatment: a Dutch population-based study," Journal of Clinical Oncology, vol. 26, no. 8, pp. 1239-1246, 2008.

[34] H. B. Muss, D. A. Berry, C. Cirrincione et al., "Toxicity of older and younger patients treated with adjuvant chemotherapy for node-positive breast cancer: the Cancer and Leukemia Group B experience," Journal of Clinical Oncology, vol. 25, no. 24, pp. 3699-3704, 2007.

[35] D. A. Patt, Z. Duan, S. Fang, G. N. Hortobagyi, and S. H. Giordano, "Acute myeloid leukemia after adjuvant breast cancer therapy in older women: understanding risk," Journal of Clinical Oncology, vol. 25, no. 25, pp. 3871-3876, 2007.

[36] D. Hershman, A. I. Neugut, J. S. Jacobson et al., "Acute myeloid leukemia or myelodysplastic syndrome following use of granulocyte colony-stimulating factors during breast cancer adjuvant chemotherapy," Journal of the National Cancer Institute, vol. 99, no. 3, pp. 196-205, 2007.

[37] A. Rossi, G. Colantuoni, P. Maione et al., "Chemotherapy of breast cancer in the elderly," Current Medicinal Chemistry, vol. 12, no. 3, pp. 297-310, 2005.

[38] G. Frasci, V. Lorusso, N. Panza et al., "Gemcitabine plus vinorelbine versus vinorelbine alone in elderly patients with advanced non-small-cell lung cancer," Journal of Clinical Oncology, vol. 18, no. 13, pp. 2529-2536, 2000.

[39] S. Rezkalla, R. A. Kloner, J. Ensley et al., "Continuous ambulatory ECG monitoring during fluorouracil therapy: a prospective study," Journal of Clinical Oncology, vol. 7, no. 4, pp. 509-514, 1989.

[40] S. Firat, M. Bousamra, E. Gore, and R. W. Byhardt, "Comorbidity and KPS are independent prognostic factors in stage I non-small-cell lung cancer," International Journal of Radiation Oncology Biology Physics, vol. 52, no. 4, pp. 1047-1057, 2002.

[41] J. F. Piccirillo, R. M. Tierney, I. Costas, L. Grove, and E. L. Spitznagel, "Prognostic importance of comorbidity in a hospital-based cancer registry," Journal of the American Medical Association, vol. 291, no. 20, pp. 2441-2447, 2004.

[42] D. M. Fick, J. W. Cooper, W. E. Wade, J. L. Waller, J. R. Maclean, and M. H. Beers, "Updating the Beers criteria for potentially inappropriate medication use in older adults: results of a US consensus panel of experts," Archives of Internal Medicine, vol. 163, no. 22, pp. 2716-2724, 2003.

[43] P. J. Barry, N. O’Keefe, K. A. O'Connor, and D. O’Mahony, "Inappropriate prescribing in the elderly: a comparison of the Beers criteria and the improved prescribing in the elderly tool (IPET) in acutely ill elderly hospitalized patients," Journal of Clinical Pharmacy and Therapeutics, vol. 31, no. 6, pp. 617626, 2006. 
[44] L. Ferrucci, J. M. Guralnik, C. Cavazzini et al., "The frailty syndrome: a critical issue in geriatric oncology," Critical Reviews in Oncology/Hematology, vol. 46, no. 2, pp. 127-137, 2003.

[45] M. Extermann, "Studies of comprehensive geriatric assessment in patients with cancer," Cancer Control, vol. 10, no. 6, pp. 463-468, 2003.

[46] M. Extermann and A. Hurria, "Comprehensive geriatric assessment for older patients with cancer," Journal of Clinical Oncology, vol. 25, no. 14, pp. 1824-1831, 2007.

[47] A. Hurria, S. Gupta, M. Zauderer et al., "Developing a cancerspecific geriatric assessment: a feasibility study," Cancer, vol. 104, no. 9, pp. 1998-2005, 2005.

[48] H. J. Cohen, J. R. Feussner, M. Weinberger et al., "A controlled trial of inpatient and outpatient geriatric evaluation and management," New England Journal of Medicine, vol. 346, no. 12, pp. 905-912, 2002.

[49] L. Balducci, H. J. Cohen, P. Engstrom et al., "Senior adult oncology clinical practice guidelines in oncology," JNCCN Journal of the National Comprehensive Cancer Network, vol. 3, no. 4, pp. 572-590, 2005.

[50] T. Wasil and S. M. Lichtman, "Clinical pharmacology issues relevant to the dosing and toxicity of chemotherapy drugs in the elderly," Oncologist, vol. 10, no. 8, pp. 602-612, 2005.

[51] S. M. Bond, "Physiological aging in older adults with cancer: implications for treatment decision making and toxicity management," Journal of Gerontological Nursing, vol. 36, no. 2, pp. 26-39, 2010.

[52] J. Walston, E. C. Hadley, L. Ferrucci et al., "Research agenda for frailty in older adults: toward a better understanding of physiology and etiology: summary from the American Geriatrics Society/National Institute on Aging research conference on frailty in older adults," Journal of the American Geriatrics Society, vol. 54, no. 6, pp. 991-1001, 2006.

[53] I. Carreca and L. Balducci, "Cancer chemotherapy in the older cancer patient," Urologic Oncology, vol. 27, no. 6, pp. 633-642, 2009.

[54] D. N. Pearlman, L. G. Branch, R. J. Ozminkoivski, B. Experton, and $\mathrm{Z}$. Li, "Transitions in health care use and expenditures among frail older adults by payor/provider type," Journal of the American Geriatrics Society, vol. 45, no. 5, pp. 550-557, 1997.

[55] X. Yao, H. Li, and S. X. Leng, "Inflammation and immune system alterations in frailty," Clinics in Geriatric Medicine, vol. 27, no. 1, pp. 79-87, 2011.

[56] D. C. Dale, "Poor prognosis in elderly patients with cancer: the role of bias and undertreatment," The Journal of Supportive Oncology, vol. 1, no. 4, supplement 2, pp. 11-17, 2003.

[57] G. H. Lyman, "Caring for the elderly cancer patient: training the next generation of oncologists," Journal of Oncology Practice, vol. 4, no. 4, pp. 193-194, 2008. 


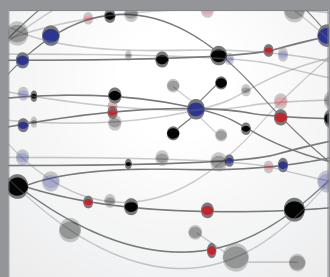

The Scientific World Journal
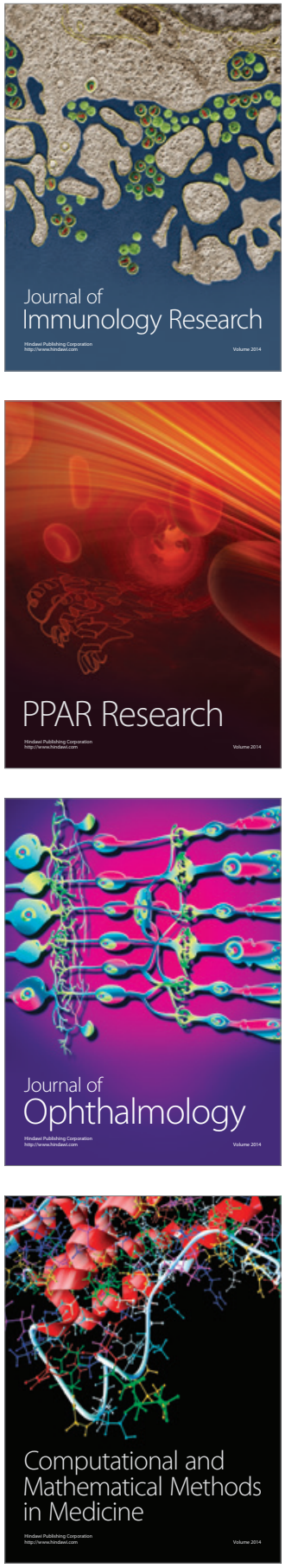

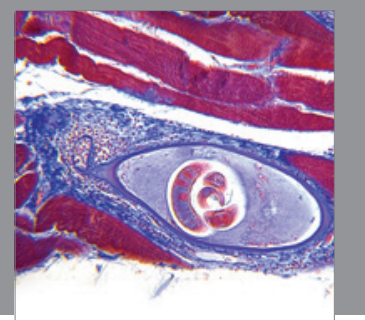

Gastroenterology

Research and Practice
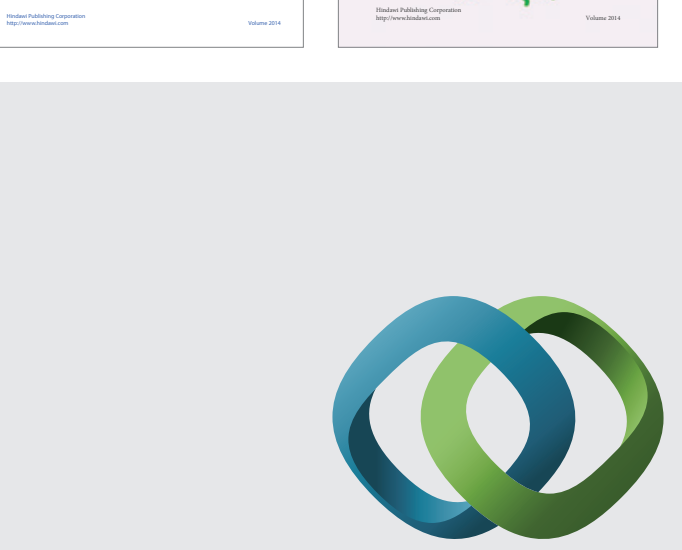

\section{Hindawi}

Submit your manuscripts at

http://www.hindawi.com
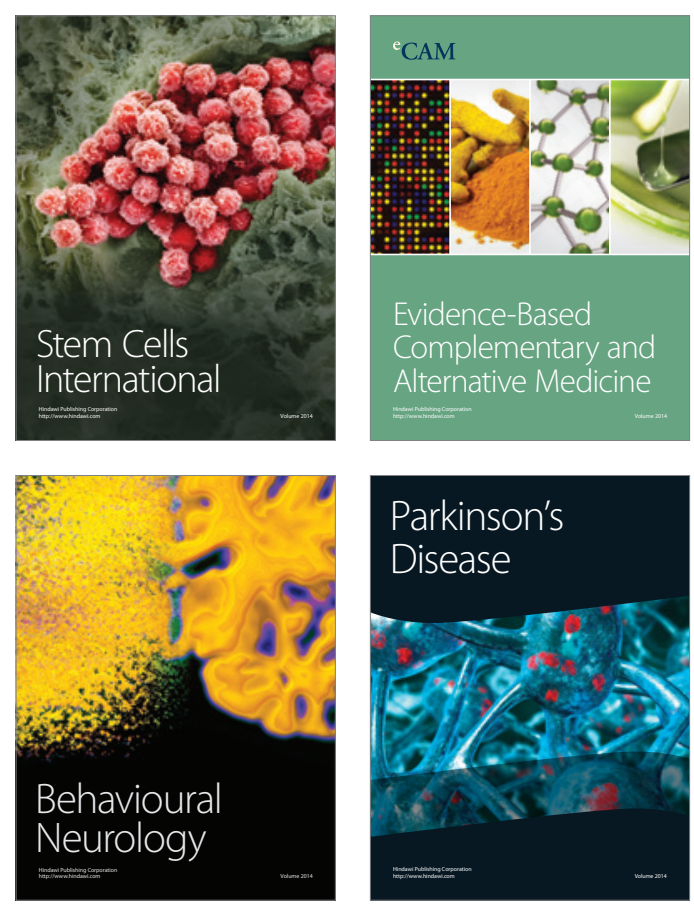

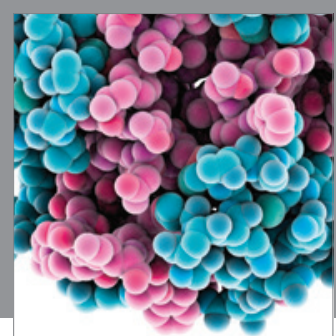

Journal of
Diabetes Research

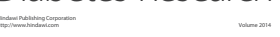

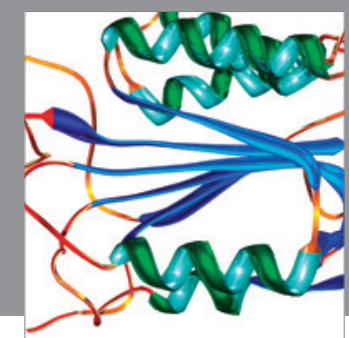

Disease Markers
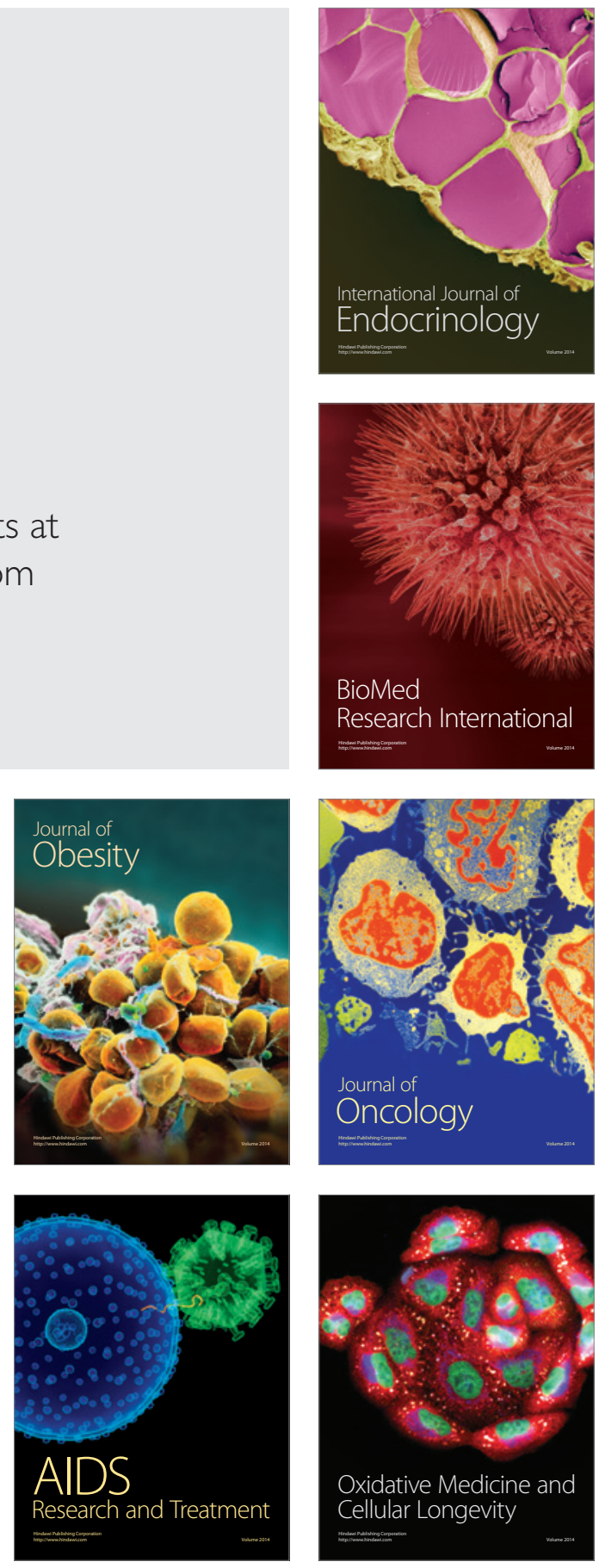